Handwritten al phabet and di git char acter recogni ti on usi ng skel et on pat ter $n$ mappi ng wi th st ructural const rai nt s

\begin{tabular}{|c|c|}
\hline 著者 & Nakay ana Kenj i, Hasegawa H., Her nandez C. A. \\
\hline $\begin{array}{l}\text { journal or } \\
\text { publ i cat } i \text { on } t i t l e\end{array}$ & $\begin{array}{l}\text { I cann ' } 93 \text { : Proceedi ngs of the I nt er nat i onal } \\
\text { Conf er ence on Arti fi ci al Neur al Net works } \\
\text { Anst er dam the Net her I ands }\end{array}$ \\
\hline page $r$ ange & 941 \\
\hline year & $1993-09-01$ \\
\hline URL & ht t p: //hdl . handl e. net /2297/18742 \\
\hline
\end{tabular}




\title{
HANDWRITTEN AIPIABET AND DIGIT CHARACTER RECOGNITION USING SKELETON PATTERN MAPPING WITH STRUCTURAL CONSTRAINTS
}

\section{KenjiNAKAYAMA Osamu HASEgAWA Carlos HERNANDEZ E. ${ }^{+}$}

\author{
Dept. of Electrical \& Computer Eng., Kanazawa Univ. Japan \\ E-mıil: nakayama@haspnn1.ec.t.kanazawa-u.ac.jp \\ +Computers \& Electronics Dept., Valencia Univ. Spain
}

\begin{abstract}
This paper presents a handwritten character recognition method, which can be applied to highly distorted patterns, including bold lines, non-uniform line width and blurred lines. Complicated pre-processing is not required. Distorted patterns $\mathrm{Q}(\mathrm{m})$ can be directly applied to this system. A skeleton pattern $S(n)$ of standard patterns $P(n)$ is employed to analyze structure of $Q(m)$. $S(n)$ is mapped onto $Q(m)$. while conserving pattern structure. If $\mathrm{P}(\mathrm{n})$ has different structure from that of $\mathrm{Q}(\mathrm{m})$, then it cannot be exactly mapped onto $Q(\mathbf{m})$. Thus, this approach realizes "mental distortion" like in the human visual system. As the mapping makes progress, structural constraints are gradually relayed. At the same time, some partial structures are periodically imposed. Fur thermore, redundant points are removed from $S(n)$, or extra points are added to $S(n) . Q(m)$ is recognized based on consistency between $Q(m)$ and the mapped version of $S(n)$.
\end{abstract}

\section{INTRODUCTION}

Handwritten character recognition is very important application fields of neural networks. Neocognitron is the first neural model, which can be applied to distorted pattern recognition [1]. Recently, many approaches, based on multilayer neural networks, modified back-propagation and self-organizing methods have been proposed [2]-[6]. Some of them extract invariant features, and apply them to pattern matching using neural networks. In another methods, the distorted patterns are directly applied to the neural networks, in which feature extraction and pattern matching are simultaneously carried out. The authors have developed locally connected neural networks to extract node and line features, and modified self-organizing feature map. They have been combined to perform handwritten character recognition [7].

Several difficulties still remain in handwritten character recognition. Scaling and translation of patterns can be compensated for. However, rotation and high distortions including bold lines, non-uniform line width and blurred lines still cause some limitations. In this paper, a character recognition method is proposed, which is robust for highly distorted patterns.

\section{HANDWRITTEN CIIARACTER RECOGNITION}

\subsection{Basic Strategy}

In this paper, handwritten alphabet and digit characters, having bold lines, non-uniform line width and blurred lines, are taken into account. Distorted patterns can be directly applied to the proposed system. It does not require complicated pre-processing. Basic strategy follows human brain like processing, that is "mental distortion". The process is based on pattern mapping from standard to distorted patterns. However, essential structure information is conserved during the mapping. This process is based on Kohonen's self-organizing feature map (SOM) [8]. However, several improvements are proposed in order to apply it to highly distorted pattern recognition. 


\subsection{Recognition Process}

A set of standard patterns $P(n)$ is prepared. Skeletal structure is extracted, and stored in advance. Let $S(n)$ be the skeleton pattern for $P(n)$. It consists of points and lines connecting them along the pattern. Thus, the lines represent the neighbor relations between two points. Let a handwritten version of $P(n)$ be $\mathrm{Q}(\mathrm{m})$. The recognition process is summarized in the following.

(1) The distorted pattern $\mathrm{Q}(\mathrm{m})$ to be recognized is applied to the system.

(2) Appropriate standard patterns are selected based on pixel reduction patterns of $Q(m)$ and $P(n)$. At this step, exact selection is not necessary.

(3) $S(n)$ for the selected $P(n)$ is mapped onto $Q(m)$, while conserving pattern structure. This process is repeated for all selected standard patterns.

(4) Let $S_{F}(n)$ be $S(n)$ after the mapping. Consistency between $S_{F}(n)$ and $Q(m)$ is estimated. The distorted pattern is recognized based on this consistency.

\section{SKELETON PATTERN MAPPING WITH STRUCTURAL CONSTRAINTS}

\subsection{Mapping Algorithm}

$\mathrm{S}(\mathrm{n})$ is further expressed as follows:

$$
\begin{aligned}
& S(n)=\{s(n, i), l(n, i, j)\} \\
& s(n, i)=\left[s(n, 1), s(n, 2), \ldots, s\left(n, N_{s n}\right)\right] \\
& l(n, i, j)=[l(n, 1,2), 1(n, 1,3), \ldots]
\end{aligned}
$$

$s(n, i)$ is the ith point in $S(n) . l(n, i, j)$ is a line connecting the ith point and the $j$ th point in $S(n) . Q(m)$ is also expressed as

$$
Q(m)=\{q(m, i)\}, i=1,2, \ldots, N a m
$$

$q(m, i)$ is the ith pixel included in the distorted pattern $Q(m)$. The mapping process is described in the following.

(1) One of $q(m, i), i=1,2, \ldots, N_{a m}$ is randomly selected. Let this point be $q(m, i)$ for instance. $s(n, k)$ is selected, which locates most close to $q(m, i)$.

(2) $s(n, k)$ is shifted toward $q(m, 1)$ by distance $d_{\circ}$. At the same time, the first neighbor points of $s(n, k)$ are shifted toward the same direction by distance $d_{1}($ $\left.<d_{\square}\right)$. Furthermore, the second neighbor points are also shifted. The neighbor points can be detected using the lines $l(n, i, j)$. By this way, the structure of the standard pattern $\mathrm{P}(\mathrm{n})$ can be conserved.

(3) Steps (1) and (2) are repeated for all $\mathrm{q}(\mathrm{m}, \mathrm{i})$ many times by relaxing structural constraints gradually.

(4) During the mapping, partial structure constraints, which are common in actual handwritten characters, are imposed periodically.

(5) After the mapping reaches to some steady state, the mapped $S(n)$ is investigated. If redundant points exist outside $Q(m)$, then they are removed from $S(n)$. On the other hand, if the distribution of $u(n, k)$ is sparse in some parts of $Q(m)$, then extra points are added to $S(n)$. Letting the modified skeleton pattern be $S_{R}(n)$, it is further mapped onto $Q(m)$ in the same way as in (1) and (2).

(6) Let the final skeleton pattern be $S_{F}(n)$, and its points be $s_{F}(n, i)$. Consistency between $S_{F}(n)$ and $Q(m)$ is estimated by

$$
\begin{aligned}
& \mathrm{C}_{1}\left[\mathrm{Q}(\mathrm{m}), \mathrm{S}_{F}(\mathrm{n})\right]=-\alpha \sum_{\mathrm{i}=1}^{\mathrm{N}_{\mathrm{Om}}} \mathrm{d}_{\mathrm{a}}(\mathrm{m}, \mathrm{r}) \\
& \mathrm{C}_{2}\left[\mathrm{Q}(\mathrm{m}), \mathrm{S}_{F}(\mathrm{n})\right]=-\beta \sum_{\mathrm{k}-1}^{N_{S F}} \mathrm{~d}_{B}(\mathrm{n}, \mathrm{k})
\end{aligned}
$$

$\left.d_{a(m .} n\right)$ is distance between $q(m, i)$ and the nearest $s(n, k) . d_{s(n, k)}$ is length of a 
part of a strait line connecting the neighbor points. This part does not locate on $\mathrm{Q}(\mathrm{m}) . \alpha$ and $\beta$ are weighting factors determined by relation between $\mathrm{Nam}_{\mathrm{m}}$ and $N_{S F}$. If the points $S_{F}(n, k)$ are uniformly distributed over $Q(m), C_{1}$ can take the minimum value. If all lines connecting the adjoining points, are included in $Q(\mathrm{~m})$, then $\mathrm{C}_{\boldsymbol{z}}$ becomes zero. Furthermore, the number of points removed from $\mathrm{S}(\mathrm{n})$ during the mapping is also regarded as negative estimation.

\subsection{Adaptive Modification of Skeleton Pattern}

It is desirable to reduce the number of standard patterns. It is, however, very complicate to find $S(n)$, which can be commonly used. Therefore, the adaptive modifications of $S(n)$ are proposed. In the handwritten characters, the lengths of some parts of lines are easily changed. This causes redundant points or lack of points in $S(n)$. When some parts of $Q(m)$ are shortened, some $s(n, k)$ are not effectively used. These redundant points are removed. The end points are always used. On the other hand, the lines of $Q(\mathrm{~m})$ are extended, some points are inserted in $S(n)$. This modification does not affect the pattern structure.

Gradual relaxation of structure constraints is necessary in order to adapt $\mathrm{S}(\mathrm{n})$ to $\mathrm{Q}(\mathrm{m})$. However, this allows different characters, having similar structure, to be exactly mapped onto $\mathrm{Q}(\mathrm{m})$, resulting in misjudgement. For this reason, partial structure constraints, which are common in actual handwritten characters, are periodically imposed in the mapping process.

\section{SIMULATION}

Handwritten alphabet and digit are used for computer simulation. The patterns are expressed with $32 \times 32$ pixels. The mapping is carried out on $256 \times 256$ grids in order to achieve continuous shifting of the skeleton patterns.

Figure 1 shows the simulation results. The distorted pattern for ${ }^{2} 5 \mathrm{~s}$ is used. Standard patterns ${ }^{\prime} 5 \mathrm{~s}$ and ${ }^{\prime} 6 \mathrm{~s}$ are investigated. Symbols " $\mathrm{O}$ " and " . " on the plane correspond to $s(n, k)$ and $q(m, i)$, respectively. Figures $(x 1), x=a$ and $b$ are the initial states, (x2) are the middle states just before removing the redundant points, and (x3) are the final states. Since the standard patterns $[5$, has the same structure, its skeleton pattern can be exactly mapped onto the distorted one. On the other hand, the pattern of $[6]$ has different structure. Therefore, the mapping is incomplete. Namely, one line exits outside the distorted pattern. The consistency $C_{1}$ for ${ }^{\prime} 5$, is higher than that for $r 6$ s by $22 \%$. The other consistency $\mathrm{C}_{2}$ becomes zero and negative for $[5\rfloor$ and $[6\rfloor$, respectively.

Figure 2 shows another example. The distorted version of $\mathrm{r}_{\mathrm{M}} \mathrm{s}$ and standard patterns ${ }^{r} \mathrm{H} s$ and ${ }^{2} M_{s}$ are investigated. Although the skeleton pattern of ${ }^{r} \mathrm{H} s$ can be mapped onto the distorted one, all lines cannot be included in it. $R e-$ garding the consistencies $\mathrm{C}_{1}$ and $\mathrm{C}_{2}$, almost same results are obtained.

\section{CONCLUSIONS}

The handwritten character recognition method, which employs the skeleton pattern mapping with structure constraints, has been proposed. Ilighly distorted alphabet and digit characters can be recognized.

\section{REFERENCES}

[1]K. Fukushima, S.Miyake and T.Ito, "Neocognitron: A neural network model for a mechanism of visual pattern recognition", IEEE Trans. vol.SMC-13, pp.826-834, 1983.

[2]Y. Chen et al., "Backpropagation applied to handwritten zip code recognition", Neural Network, vol. 1, no.4, pp.541-551, 1989.

[3]Y.Hirai and Y.Tsukui, "Position independent neuro pattern matching and Its application to handwritten numeral character recognition", Proc. IJCNN' 91 San Diego, pp. III-695-701, 1991.

[4]T.P.Vogl et al., "Classification of hand-written digits and Japanese Kanj1", Proc. IJCNN'91 
Seattle, pp. I -97-102, 1991.

[5]S.Lee and J.Pan, "Handwritten numeral recognition based on hierarchically self-organizing learning networks", Proc. IJCNN'91 Singapore, pp.1313-1322, 1991.

[6]Y.Idan and R.C.Chevallen, "Handwritten digits recognltion by a supervised Kohonen-like learning algorithm", Proc. IJCNN'91 Singapore, pp.2576-2581, 1991.

[7]K. Nakayama Y.Chlgawa and 0.Hasegawa, "Handwritten alphabet and digit character recognitlon using feature extracting neural network and modifled self-organizing map", Proc. IJCNN'92 Baltimore, pp. IV-235-239 June 1992.

[8]T.Kohonen, "The self-organizing map", Proc. IEEE, vol.78, no.9, pp.1464-1480, Sept. 1990.
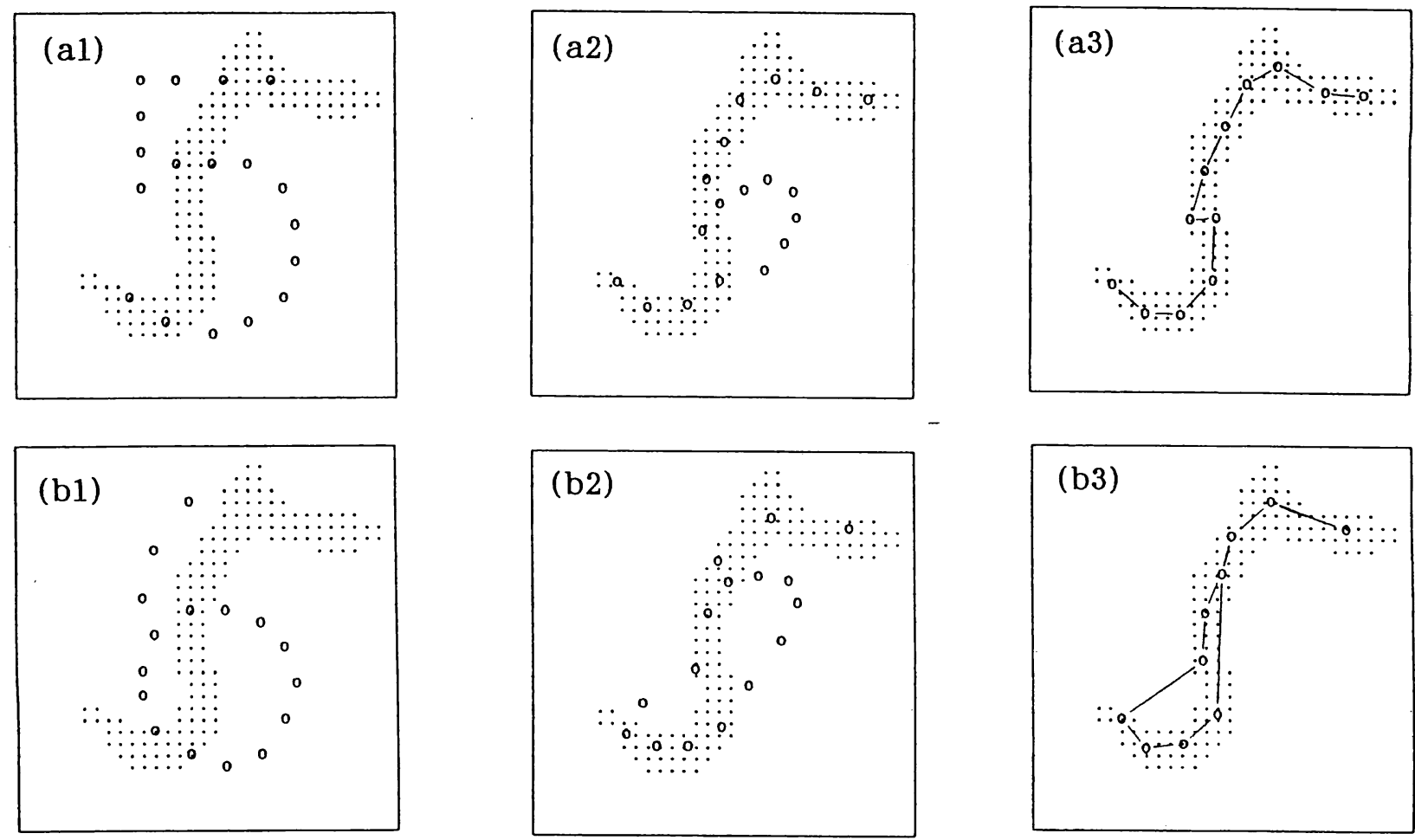

Fig. 1 Skeleton pattern mapping using distorted ' 5 」, and standard $\lceil 5$ 」 and ' 6 」.
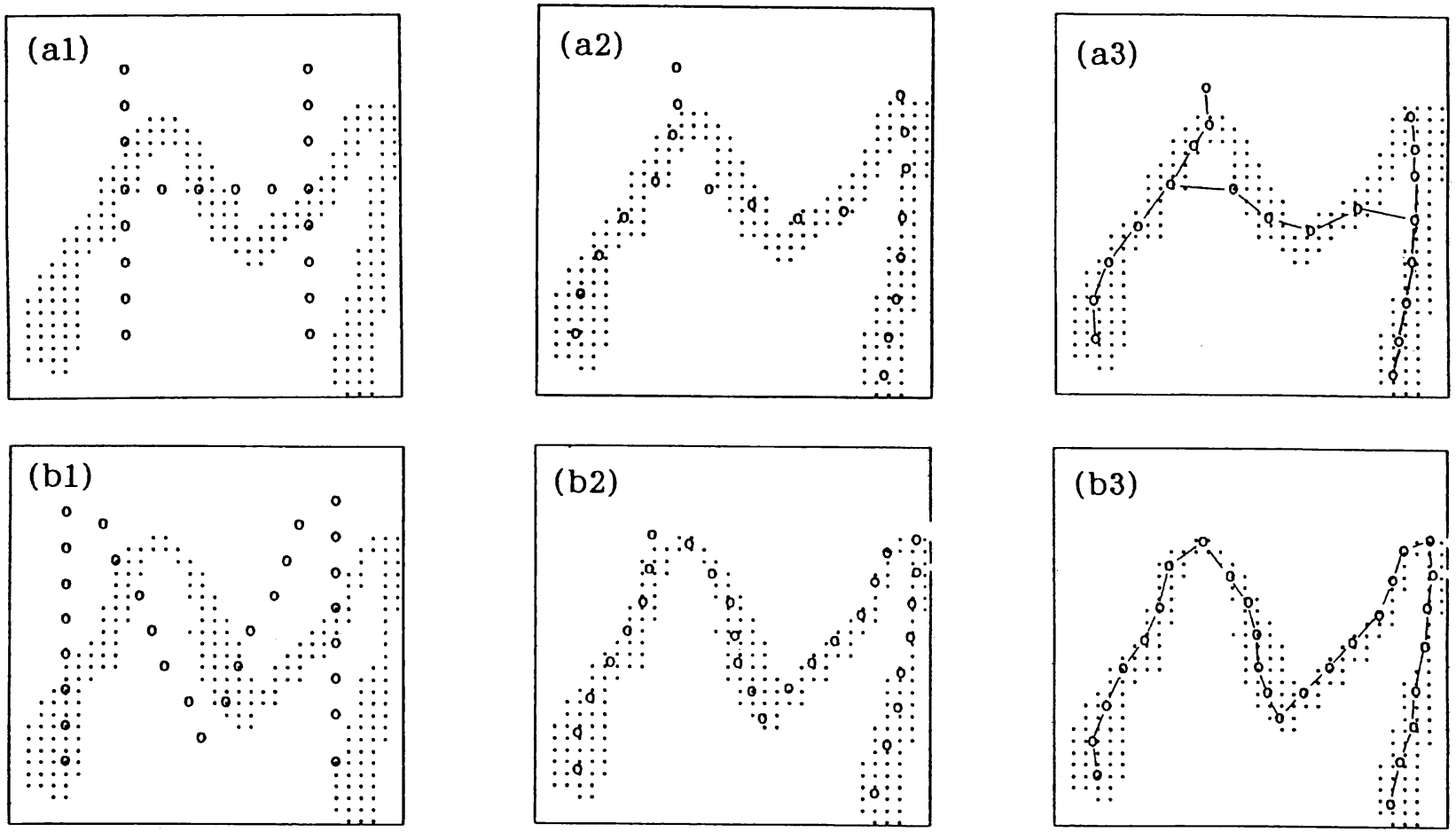

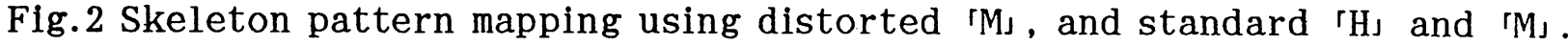

